\title{
Ginsenoside Rh2 Inhibits Cancer Stem-Like Cells in Skin Squamous Cell Carcinoma
}

\author{
Shunli Liu ${ }^{a}$ Mingrui Chen ${ }^{a}$ Pengcheng Li ${ }^{b}$ Yujia Wu Chunjuan Chang ${ }^{b}$ \\ Yabin Qiu ${ }^{b}$ Ling Cao ${ }^{b}$ Zhen Liu ${ }^{b}$ Chiyu Jiab \\ aDepartment of Burns and Plastic Surgery, General Hospital of Jinan Military Region, Jinan, \\ ${ }^{b}$ Department of Burns and Plastic Surgery, the 309th Hospital of PLA, Beijing, China
}

\section{Key Words}

Skin squamous cell carcinoma (SCC) • Cancer stem cells (CSCs) • Lgr5 • Ginsenoside Rh2 $(\mathrm{GRh} 2) \cdot \beta$-catenin

\begin{abstract}
Background/Aims: Treatments targeting cancer stem cells (CSCs) are most effective cancer therapy, whereas determination of CSCs is challenging. We have recently reported that Lgr5positive cells are cancer stem cells (CSCs) in human skin squamous cell carcinoma (SCC). Ginsenoside Rh2 (GRh2) has been shown to significantly inhibit growth of some types of cancers, whereas its effects on the SCC have not been examined. Methods: Here, we transduced human SCC cells with lentivirus carrying GFP reporter under Lgr5 promoter. The transduced SCC cells were treated with different doses of GRh2, and then analyzed cell viability by CCK8 assay and MTT assay. The effects of GRh2 on Lgr5-positive CSCs were determined by flow cytometry and by tumor sphere formation. Autophagy-associated protein and $\beta$-catenin were measured by Western blot. Expression of short hairpin small interfering RNA (shRNA) for Atg7 and $\beta$-catenin were used to inhibit autophagy and $\beta$-catenin signaling pathway, respectively, as loss-of-function experiments. Results: We found that GRh2 dose-dependently reduced SCC viability, possibly through reduced the number of Lgr5-positive CSCs. GRh2 increased autophagy and reduced $\beta$-catenin signaling in SCC cells. Inhibition of autophagy abolished the effects of GRh2 on $\beta$-catenin and cell viability, while increasing $\beta$-catenin abolished the effects of GRh2 on autophagy and cell viability. Conclusion: Taken together, our data suggest that GRh2 inhibited SCC growth, possibly through reduced the number of Lgr5-positive CSCs. This may be conducted through an interaction between autophagy and $\beta$-catenin signaling.
\end{abstract}

Zhen Liu, and Chiyu Jia

KARGER 125
Department of Burns and Plastic Surgery, The 309th Hospital of PLA, 17 Heishanhu Street, Beijing 100091 (China)

E-Mail zddm329@126.com; E-Mail 133911238533@163.com 


\section{Introduction}

Carcinoma account for more than $80 \%$ of all types of cancer worldwide, and squamous cell carcinoma (SCC) is the most frequent carcinoma. Skin SCC causes a lot of mortality yearly, which requires a better understanding of the molecular carcinogesis of skin SCC for developing efficient therapy [1,2]. Ginsenoside Rh2 (GRh2) is a characterized component in red ginseng, and has proven therapeutic effects on inflammation [3] and a number of cancers [4-14], whereas its effects on the skin SCC have not been examined.

Cancer stem cells (CSCs) are cancer cells with great similarity to normal stem cells, e.g., the ability to give rise to various cell types in a particular cancer $[15,16]$. CSCs are highly tumorigenic, compared to other non-CSCs. CSCs appear to persist in tumors as a distinct population and CSCs are believed to be responsible for cancer relapse and metastasis after primary tumor resection [15-18]. Recently, the appreciation of the critical roles of CSCs in cancer therapy have been continuously increasing, although the identification of CSCs in a particular cancer is still challenging.

To date, different cell surface proteins have been used to isolate CSCs from a variety of cancers by flow cytometry. Among these markers for identification of CSCs, the most popular ones are prominin-1 (CD133), side population (SP) and increased activity of aldehyde dehydrogenase (ALDH). CD133 is originally detected in hematopoietic stem cells, endothelial progenitor cells and neuronal and glial stem cells. Later on, CD133 has been shown to be expressed in the CSCs from some tumors [19-23], but with exceptions [24]. SP is a sub-population of cells that efflux chemotherapy drugs, which accounts for the resistance of cancer to chemotherapy. Hoechst (HO) has been experimentally used for isolation of SP cells, while the enrichment of CSCs by SP appears to be limited [25]. Increased activity of ALDH, a detoxifying enzyme responsible for the oxidation of intracellular aldehydes [26,27], has also been used to identify CSCs, using aldefluor assay [28, 29]. However, ALDH has also been detected in other cell types, which creates doubts on the purity of CSCs using ALDH method [30,31]. Moreover, all these methods appear to be lack of cancer specificity.

The Wnt target gene Lgr5 has been recently identified as a stem cell marker of the intestinal epithelium, and of the hair follicle $[32,33]$. Recently, we reported that Lgr5 may be a potential CSC marker for skin SCC [34]. We detected extremely high Lgr5 levels in the resected skin SCC specimen from the patients. In vitro, Lgr5-positive SCC cells grew significantly faster than Lgr5-negative cells, and the fold increase in growth of Lgr5-positive vs Lgr5-negative cells is significantly higher than SP vs non-SP, or ALDH-high vs ALDH-low, or CD133-positive vs CD133-negative cells. Elimination of Lgr5-positive SCC cells completely inhibited cancer cell growth in vitro.

Here, we transduced human SCC cells with lentivirus carrying GFP reporter under Lgr5 promoter. The transduced SCC cells were treated with different doses of GRh2, and then analyzed cell viability by CCK-8 assay and MTT assay. The effects of GRh2 on Lgr5-positive CSCs were determined by flow cytometry and by tumor sphere formation. Autophagyassociated protein and $\beta$-catenin were measured by Western blot. Expression of short hairpin small interfering RNA (shRNA) for autophagy-related protein 7 (Atg7) and $\beta$-catenin were used to inhibit autophagy and $\beta$-catenin signaling pathway, respectively, as loss-offunction experiments. Atg7 was identified based on homology to Pichia pastoris GSA7 and Saccharomyces cerevisiae APG7. In the yeast, the protein appears to be required for fusion of peroxisomal and vacuolar membranes. The protein shows homology to the ATP-binding and catalytic sites of the E1 ubiquitin activating enzymes. Atg7 is a mediator of autophagosomal biogenesis, and is a putative regulator of autophagic function [35-38]. We found that GRh2 dose-dependently reduced SCC viability, possibly through reduced the number of Lgr5positive CSCs. GRh2 increased autophagy and reduced $\beta$-catenin signaling in SCC cells. Inhibition of autophagy abolished the effects of GRh2 on $\beta$-catenin and cell viability, while increasing $\beta$-catenin abolished the effects of GRh2 on autophagy and cell viability. 


\section{Cellular Physiology Cell Physiol Biochem 2015;36:499-508 \begin{tabular}{l|l} 
and Biochemistry Published online: May 11, 2015 & $\begin{array}{l}\text { C 2015 S. Karger AG, Basel } \\
\text { www.karger.com/cpb }\end{array}$ \\
\hline
\end{tabular}}

Liu et al.: GRh2 Inhibits CSCs in SCC

\section{Materials and Methods}

\section{Cell line and GRh2 in vitro administration}

A431 cells are a human skin SCC cell line, which was purchased from American Type Culture Collection (ATCC, Rockville, MD, USA), and cultured in Dulbecco's modified Eagle's medium (DMEM, Invitrogen, Carlsbad, CA, USA) supplemented with 15\% fetal bovine serum (FBS; Sigma-Aldrich, St Louis, MO, USA) in a humidified chamber with $5 \% \mathrm{CO}_{2}$ at $37^{\circ} \mathrm{C}$, as has been described before [34]. GRh2 (Weikeqi Bioscience, China) was prepared in a stock of $100 \mathrm{mg} / \mathrm{ml}$ and applied to cultured GBM cells at $0.01 \mathrm{mg} / \mathrm{ml}, 0.1 \mathrm{mg} / \mathrm{ml}$ and $1 \mathrm{mg} / \mathrm{ml}$, respectively.

\section{Lentivirus preparation, cell transfection and cell transduction}

Plasmids carrying GFP reporter under Lgr5 promoter were prepared as has been described before [34]. The coding sequence of human Lgr5 promoter was amplified using human genomic DNA as a template, and cloned into pLVX-ZsGreen1-C1 vector (Clontech, Mountain View, CA, USA). The coding sequence of human $\beta$-catenin was amplified using human liver cDNA as a template, and cloned into pLVXZsGreen1-C1 vector. The shRNA for autophagy-related protein 7 (Atg7) was purchased from Qiagen (Hilden, Germany). A scramble sequence was used as the mock control (scr). To generate pLgr5-GFP lentiviral particles, NIH HEK293T cells were seeded in a $100 \mathrm{~mm}$ dish at 50,000 cells $/ \mathrm{cm}^{2}$ and co-transfected with $10 \mu \mathrm{g}$ of recombinant DNA plasmids and $5 \mu \mathrm{g}$ each of packaging plasmids (REV, pMDL and VSV-G) using Lipofectamine-2000 (Invitrogen). The supernatant containing lentiviral particles was collected 48 hours after transfection and filtered through a $0.45 \mu \mathrm{m}$ syringe filter. The fibroblasts were seeded in $100 \mathrm{~mm}$ plates at 15,000 cells $/ \mathrm{cm}^{2}$ one day prior to lentiviral infection. The lentiviral particles were added along with $10 \mu \mathrm{g} / \mathrm{ml}$ polybrene (Sigma-Aldrich) to the cell culture for 24 hours. Infected cells were selected by ampicillin resistance. Transfection with either shAtg7 or $\beta$-catenin-expressing plasmids was performed with Lipofectamine-2000 (Invitrogen).

\section{Analysis of GFP by flow cytometry}

For flow cytometry analysis and sorting, Lenti-pLgr5-GFP-transduced A431 cells were analyzed by GFP direct fluorescence. Flow cytometry was performed using a FACSAria (Becton-Dickinson Biosciences, San Jose, CA, USA) flow cytometer.

\section{MTT assay}

For assay of cell viability, cells were seeded into 24 well-plate at 10000 cells per well and subjected to a Cell Proliferation Kit (MTT, Roche, Indianapolis, IN, USA), according to the instruction of the manufacturer. The MTT assay is a colorimetric assay for assessing viable cell number, taking advantage that NADPH-dependent cellular oxidoreductase enzymes in viable cells reduce the tetrazolium dye 3-(4,5-dimethylthiazol-2-yl)2,5-diphenyltetrazolium bromide (MTT) to its insoluble formazan in purple readily being quantified by absorbance value (OD) at $570 \mathrm{~nm}$. Experiments were performed 5 times.

\section{Cell counting kit-8 (CCK-8) assay}

The CCK-8 detection kit (Sigma-Aldrich) was used to measure cell viability according to the manufacturer's instructions. Briefly, cells were seeded in a 96-well microplate at a density of $5 \times 10^{4} / \mathrm{ml}^{\text {. }}$ After $24 \mathrm{~h}$, cells were treated with resveratrol. Subsequently, CCK-8 solution (20 ml/well) was added and the plate was incubated at $37^{\circ} \mathrm{C}$ for $2 \mathrm{~h}$. The viable cells were counted by absorbance measurements with a monochromator microplate reader at a wavelength of $450 \mathrm{~nm}$. The optical density value was reported as the percentage of cell viability in relation to the control group (set as $100 \%$ ).

\section{Primary Tumor Sphere Culture}

Purified tumor cells by flow cytometry were washed, acutely dissociated in oxygenated artificial cerebrospinal fluid and subject to enzymatic dissociation. Tumor cells were then resuspended in tumor sphere media (TSM) consisting of a serum-free DMEM, human recombinant EGF (20 ng/ml; Sigma-Aldrich), bFGF (20ng/ml; Sigma-Aldrich), leukemia inhibitory factor (10ng/ml; Sigma-Aldrich) and N-acetylcysteine $\left(60 \mu \mathrm{g} / \mathrm{ml}\right.$; Sigma-Aldrich), and then plated at a density of $2 \mathrm{X} 10^{6}$ cells $/ 60 \mathrm{~mm}$ plate.

\section{KARGER}




\section{Cellular Physiology Cell Physiol Biochem 2015;36:499-508 \begin{tabular}{l|l|l} 
DOI: 10.1159/000430115 & (C) 2015 S. Karger AG, Basel
\end{tabular} and Biochemistry Published online: May 11, 2015 \\ Liu et al.: GRh2 Inhibits CSCs in SCC}

\section{Western blot}

Protein was extracted from the cultured cells with RIPA lysis buffer (1\% NP40, 0.1\% Sodium dodecyl sulfate (SDS), $100 \mu \mathrm{g} / \mathrm{ml}$ phenylmethylsulfonyl fluoride, $0.5 \%$ sodium deoxycholate, in PBS) on ice. The supernatants were collected after centrifugation at $12000 \times \mathrm{g}$ at $4^{\circ} \mathrm{C}$ for $20 \mathrm{~min}$. Protein concentration was determined using a BCA protein assay kit (Bio-rad, China), and whole lysates were mixed with $4 \times$ SDS loading buffer (125mmol/l Tris-HCl, 4\% SDS, 20\% glycerol, 100mmol/l Dithiothreitol (DTT), and 0.2\% bromophenol blue) at a ratio of $1: 3$. Samples were heated at $100{ }^{\circ} \mathrm{C}$ for $5 \mathrm{~min}$ and were separated on SDSpolyacrylamide gels. The separated proteins were then transferred to a PVDF membrane. The membrane blots were first probed with a primary antibody. After incubation with horseradish peroxidase-conjugated second antibody, autoradiograms were prepared using the enhanced chemiluminescent system to visualize the protein antigen. The signals were recorded using X-ray film. Primary antibodies were rabbit anti- $\beta$ catenin, anti-Beclin, anti-Atg7, anti-LC3 and anti- $\alpha$-tubulin (Cell Signaling, San Jose, CA, USA). Secondary antibody is HRP-conjugated anti-rabbit (Jackson ImmunoResearch Labs, West Grove, PA, USA). $\alpha$-tubulin was used as protein loading controls. The protein levels were first normalized to $\alpha$-tubulin, and then normalized to control.

\section{Quantitative real-time PCR (RT-qPCR)}

Total RNA were extracted from cultured cells with RNeasy kit (Qiagen), for cDNA synthesis. Quantitative real-time PCR (RT-qPCR) was performed in duplicates with QuantiTect SYBR Green PCR Kit (Qiagen). All primers were purchased from Qiagen. Data were collected and analyzed with 2- $\Delta \Delta \mathrm{Ct}$ method for quantification of the relative mRNA expression levels. Values of genes were first normalized against $\alpha$-tubulin, and then compared to controls.

\section{Statistical analysis}

All statistical analyses were carried out using the SPSS 18.0 statistical software package. All data were statistically analyzed using one-way ANOVA with a Bonferoni correction, followed by Fisher's exact test. All values are depicted as mean \pm standard deviation from 5 individuals and are considered significant if $\mathrm{p}<$ 0.05 .

\section{Results}

Transduction of SCC cells with GFP under Lgr5 promoter

We have recently shown that Lgr5 is CSC marker for skin SCC [34]. In order to examine the role of GRh2 on SCC cells, as well as a possible effect on CSCs, we transduced human skin SCC cells A431 [34] with a lentivirus carrying GFP reporter under Lgr5 promoter (Fig. 1A). The Lgr5-positive cells were green fluorescent in culture (Fig. 1B), and could be analyzed or isolated by flow cytometry, based on GFP (Fig. 1C).

Fig. 1. Transduc tion of SCC cells with GFP under Lgr5 promoter. (A) The structure of lentivirus carrying GFP reporter under Lgr5 promoter.

The pLgr5-GFPtransduced A431 cells in culture. Lgr5-positive cells were green fluore-

A pLgr5 GFP

B

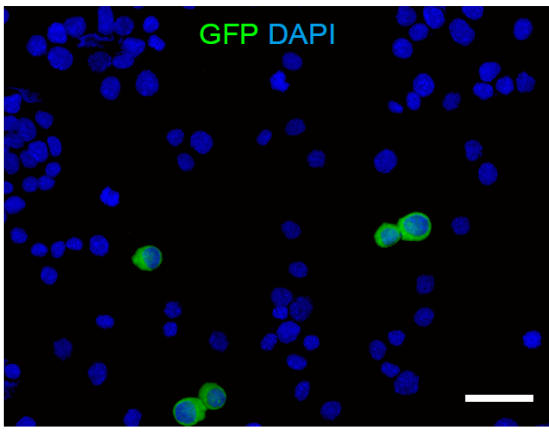

\section{C}

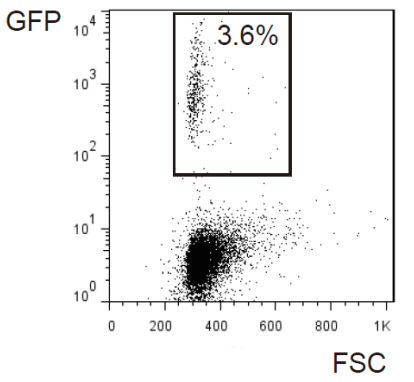

scent. Nuclear staining was done by DAPI. (C) Representative flow chart for analyzing pLgr5-GFP-transduced A431 cells by flow cytometry based on GFP. Gated cells were Lgr5-positive cells. Scar bar is $20 \mu \mathrm{m}$. 
Fig. 2. GRh2 dose-dependently inhibits SCC cell growth. We gave GRh2 at different doses $(0.01 \mathrm{mg} /$ $\mathrm{ml}, \quad 0.1 \mathrm{mg} /$ $\mathrm{ml}$ and $1 \mathrm{mg} /$ $\mathrm{ml}$ ) to the c u l t u r e d pLgr 5 -GFPtransduced A431 cells. (A-B) GRh2 dose-dependently deceased the cell viability in either a CCK8 assay (A), or a MTT assay (B). (C-D) $\mathrm{GFP}+$ cells after GRh2 treatment were analyzed by flow cytometry, showing that GRh2 dose-dependently deceased the percentage of GFP+ cells, by representative flow charts (C), and by quantification (D). The capability of the
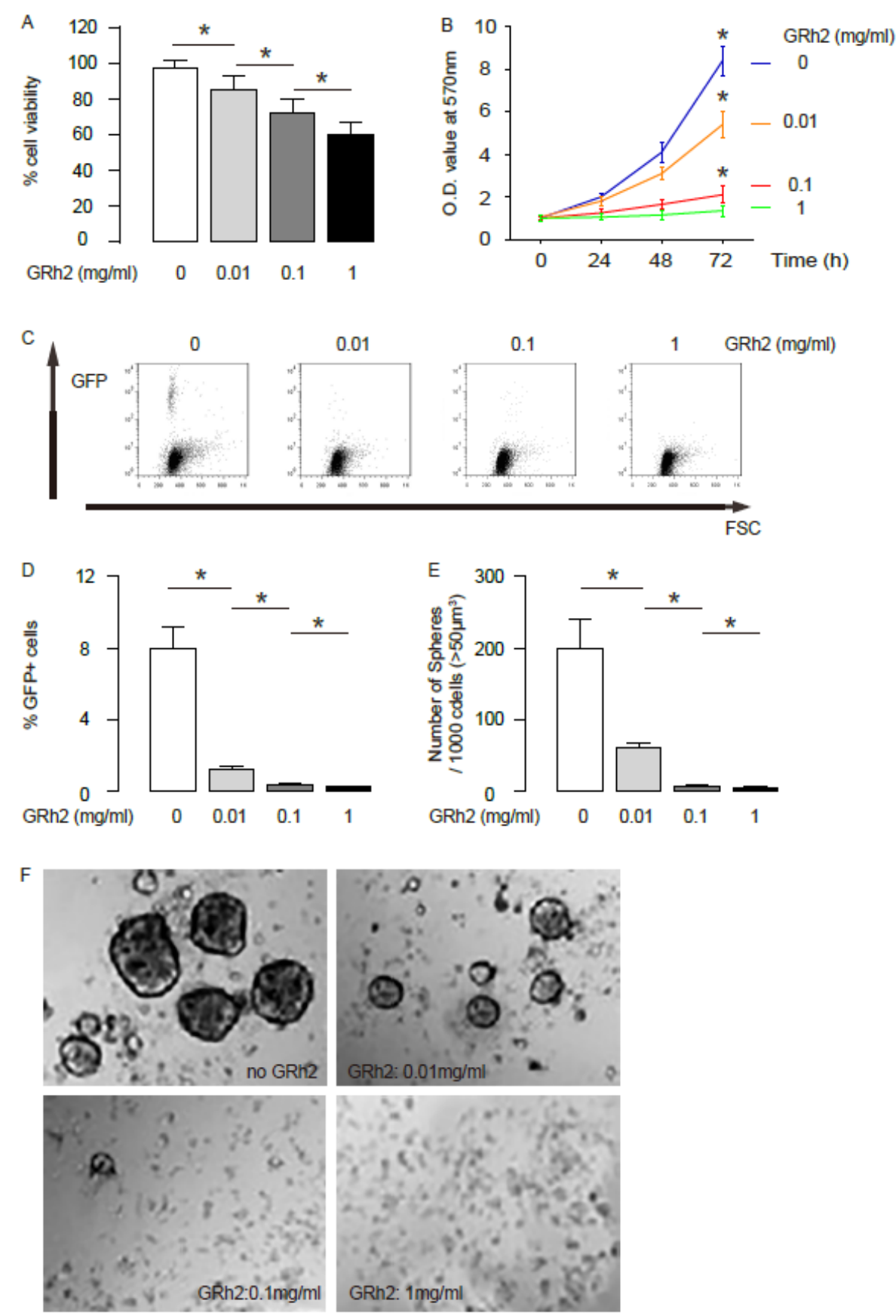

GRh2-treated

cells to form tumor sphere-like structures was examined, shown by quantification (E), and by representative images $(\mathrm{F}){ }^{*} \mathrm{p}<0.05 . \mathrm{N}=5$.

GRh2 dose-dependently inhibits SCC cell growth

Then, we examined the effect of GRh2 on the viability of SCC cells. We gave GRh2 at different doses $(0.01 \mathrm{mg} / \mathrm{ml}, 0.1 \mathrm{mg} / \mathrm{ml}$ and $1 \mathrm{mg} / \mathrm{ml})$ to the cultured pLgr5-GFP-transduced A431 cells. We found that from $0.01 \mathrm{mg} / \mathrm{ml}$ to $1 \mathrm{mg} / \mathrm{ml}$, GRh2 dose-dependently deceased the cell viability in either a CCK-8 assay (Fig. 2A), or a MTT assay (Fig. 2B). Next, we questioned whether GRh2 may have a specific effect on CSCs in SCC cells. Thus, we analyzed GFP+ cells, which represent Lgr5-positive CSCs in pLgr5-GFP-transduced A431 cells after GRh2 treatment. We found that GRh2 dose-dependently deceased the percentage of GFP+ cells, by 
Fig. 3. GRh2 treatment decreases $\beta$-catenin and increases autophagy in SCC cells. (A-B) GRh2 treatment dose-dependently decreases $\beta$-catenin, and dose-dependently upregulated autophagy-related proteins Beclin, Atg7 and increased the ratio of LC3 II to LC3 I, by quantification $(\mathrm{A})$, and by representative Western blots (B). ${ }^{*} \mathrm{p}<0.05 . \mathrm{N}=5$.
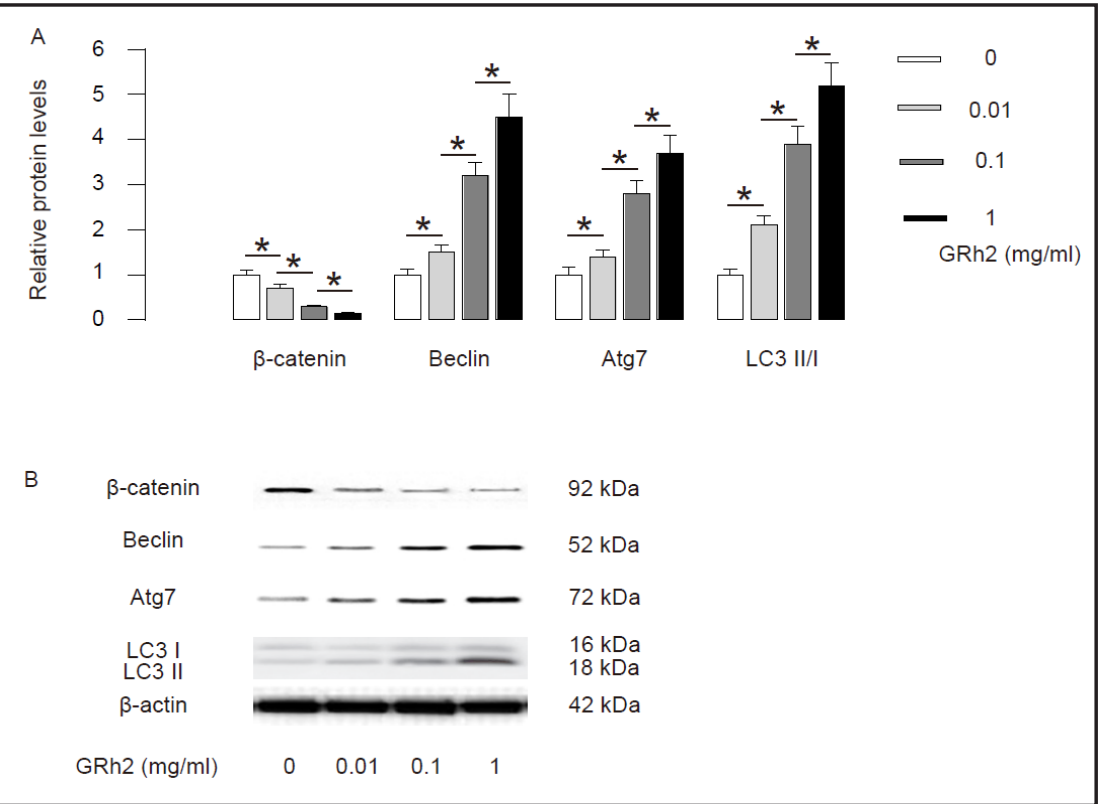

Fig. 4. Inhibition of autophagy abolishes the effects of GRh2 on $\beta$-catenin. A431 cells were transfected with shRNA for Atg7, or scrambled sequence (scr) as a control. (A) RT-qPCR for Atg7. (B) Quantification of $\beta$-catenin, Beclin, Atg7 and LC3 by Western blot. (C) Cell viability by CCK-8 assay. ${ }^{*} \mathrm{p}<0.05 . \mathrm{N}=5$.

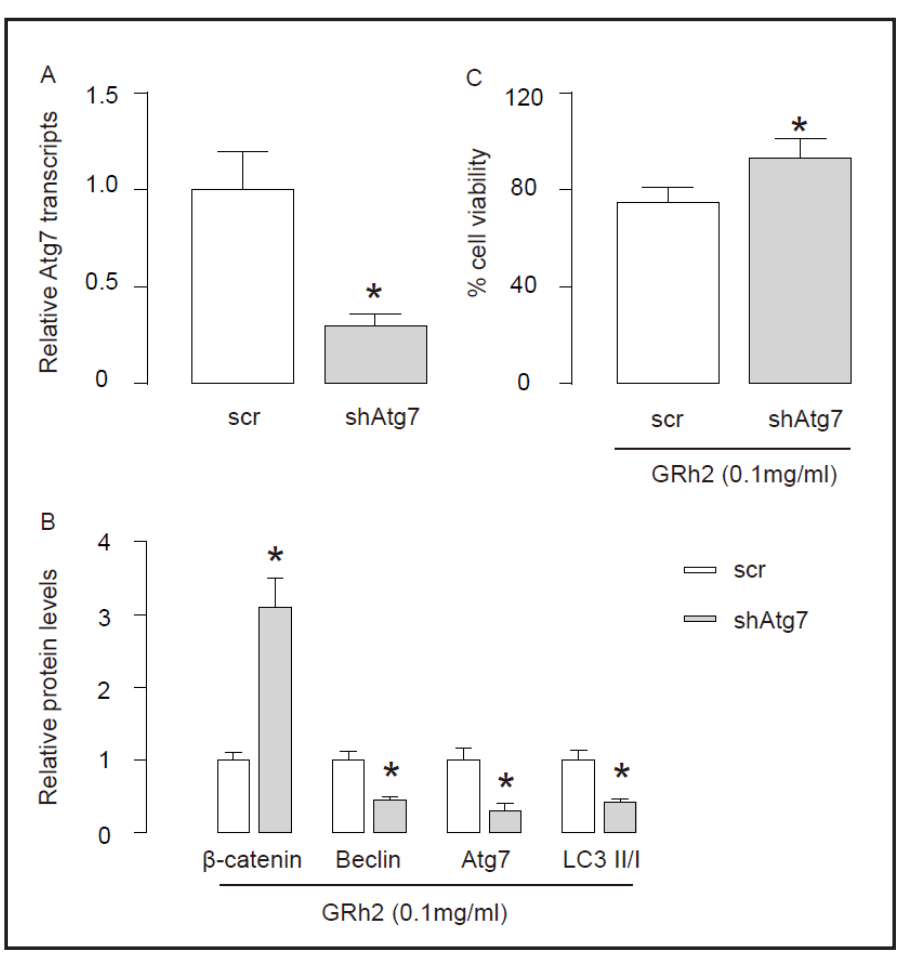

representative flow charts (Fig. 2C), and by quantification (Fig. 2D). We also examined the capability of the GRh2-treated cells in the formation of tumor sphere. We found that GRh2 dose-dependently deceased the formation of tumor sphere-like structure, by quantification (Fig. 2E), and by representative images (Fig. 2F). Together, these data suggest that GRh2 dose-dependently inhibited SCC cell growth, possibly through inhibition of CSCs.

\section{GRh2 treatment decreases $\beta$-catenin and increases autophagy in SCC cells}

We analyzed the molecular mechanisms underlying the cancer inhibitory effects of GRh2 on SCC cells. We thus examined the growth-regulatory proteins in SCC. From a variety of proteins, we found that GRh2 treatment dose-dependently decreases $\beta$-catenin, and 
Fig. 5. Overexpression of $\beta$-catenin abolishes the effects of GRh2 on autophagy. A431 cells were transfected with $\beta$-catenin, or scrambled sequence (scr) as a control. (A) RT-qPCR for $\beta$-catenin. (B) Quantification of $\beta$-catenin, Beclin, Atg7 and LC3 by Western blot. (C) Cell viability by CCK-8 assay. ${ }^{*} \mathrm{p}<0.05 . \mathrm{N}=5$.

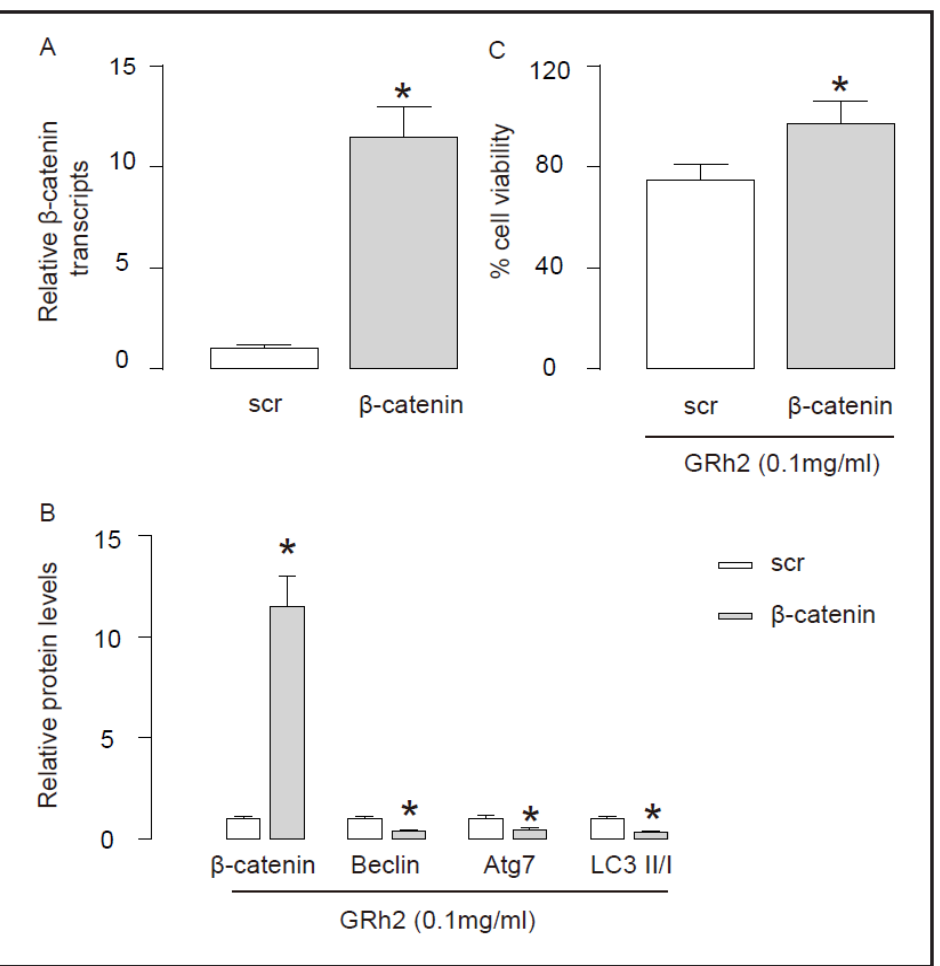

Fig. 6. Schematic of the model. GRh2 may target both $\beta$-catenin signaling and autophagy, which interacts with each other in the regulation of SCC cell viability and growth.

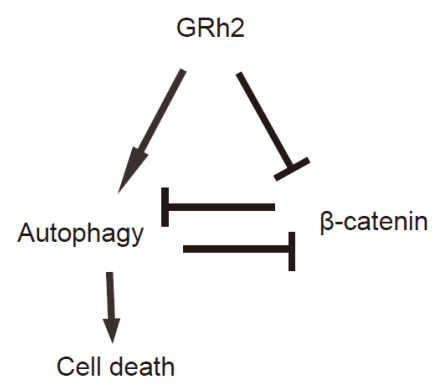

dose-dependently upregulated autophagy-related proteins Beclin, Atg7 and increased the ratio of LC3 II to LC3 I, by quantification (Fig. 3A), and by representative Western blots (Fig. 3B). Since $\beta$-catenin signaling is a strong cell-growth stimulator and autophagy can usually lead to stop of cell-growth and cell death, we feel that the alteration in these pathways may be responsible for the GRh2-mediated suppression of SCC growth.

Inhibition of autophagy abolishes the effects of GRh2 on $\beta$-catenin

In order to find out the relationship between $\beta$-catenin and autophagy in this model, we inhibited autophagy using a shRNA for Atg7, and examined its effect on the changes of $\beta$-catenin by GRh2. First, the inhibition of Atg7 in A431 cells by shAtg7 was confirmed by RT-qPCR (Fig. 4A), and by Western blot (Fig. 4B). Inhibition of Atg7 resulted in abolishment of the effects of GRh2 on other autophagy-associated proteins (Fig. 4B), and resulted in abolishment of the inhibitory effect of GRh2 on $\beta$-catenin (Fig. 4B). Moreover, the effects of GRh2 on cell viability were completely inhibited (Fig. C). Together, inhibition of autophagy abolishes the effects of GRh2 on $\beta$-catenin. Thus, the regulation of GRh2 on $\beta$-catenin needs autophagy-associated proteins.

Overexpression of $\beta$-catenin abolishes the effects of GRh2 on autophagy

Next, we inhibited the effects of GRh2 on $\beta$-catenin by overexpression of $\beta$-catenin in A431 cells. First, the overexpression of $\beta$-catenin in A431 cells was confirmed by RT-qPCR 
(Fig. 5A), and by Western blot (Fig. 5B). Overexpression of $\beta$-catenin resulted in abolishment of the effects of GRh2 on autophagy-associated proteins (Fig. 5B). Moreover, the effects of GRh2 on cell viability were completely inhibited (Fig. 5C). Together, inhibition of $\beta$-catenin signaling abolishes the effects of GRh2 on autophagy. Thus, the regulation of GRh2 on autophagy needs $\beta$-catenin signaling. This model is thus summarized in a schematic (Fig. 6 ), suggesting that GRh2 may target both $\beta$-catenin signaling and autophagy, which interacts with each other in the regulation of SCC cell viability and growth.

\section{Discussion}

Understanding the cancer molecular biology of skin SCC and identification of an effective treatment are both critical for improving the current therapy [1]. Lgr5 has been recently identified as a novel stem cell marker of the intestinal epithelium and the hair follicle, in which Lgr5 is expressed in actively cycling cells $[32,33]$. Moreover, we recently showed that Lgr5-positive are CSCs in skin SCC [34]. Thus, specific targeting Lgr5-positive cells may be a promising therapy for skin SCC.

In the current study, we analyzed the effects of GRh2 on the viability of SCC. Importantly, we not only found that GRh2 dose-dependently decreases SCC cell viability, but also dosedependently decreased the number of Lgr5-positive CSCs in SCC cells. These data suggest that the CSCs in SCC may be more susceptible for the GRh2 treatment, and the decreases in CSCs may result in the decreased viability in total SCC cells. This point was supported by following mechanism studies. Activated $\beta$-catenin signaling by WNT/GSK3 $\beta$ prevents degradation of $\beta$-catenin and induces its nuclear translocation [39]. Nuclear $\beta$-catenin thus activates c-myc, cyclinD1 and c-jun to promote cell proliferation, and activates Bcl-2 to inhibit apoptosis [39]. High $\beta$-catenin levels thus are a signature of CSCs. Therefore, it is not surprising that CSCs are more affected than other cells when GRh2 targets $\beta$-catenin signaling.

In addition, GRh2 appears to target autophagy. Although altered metabolism may be beneficial to the cancer cells, it can create an increased demand for nutrients to support cell growth and proliferation, which creates metabolic stress and subsequently induces autophagy, a catabolic process leading to degradation of cellular components through the lysosomal system [40]. Cancer cells use autophagy as a survival strategy to provide essential biomolecules that are required for cell viability under metabolic stress [40]. However, autophagy not only results in a staring in cell growth, but also may result in cell death [40]. Increases in autophagy may substantially decrease cancer cell growth. Thus, GRh2 has its inhibitory effect on skin SCC cells through a combined effect on cell proliferation (by decreasing $\beta$-catenin) and autophagy [40].

Interestingly, our data suggest an interaction between $\beta$-catenin and autophagy. This finding is consistent with previous reports showing that autophagy negatively modulates Wnt/ $\beta$-catenin signaling by promoting Dvl instability $[41,42]$, and with other studies showing that $\beta$-catenin regulates autophagy $[38,43,44]$.

Of note, we have checked other SCC lines and essentially got same results. Together with our previous reports showing that Lgr5-positive cells are CSCs in skin SCC [34], these findings thus highlight a future engagement of Lgr5-directed GRh2 therapy, which could be performed in a sufficiently frequent manner, to substantially improve the current treatment for skin SCC.

\section{Acknowledgements}

This work was supported by the National Natural Science Foundation Project (No.81401250), National Natural Science Foundation Project (No. 81372051), Medical and Senile Disease Foundation Project of General Staff Headquarters (No.ZCWS14B06), 309 Hospital of PLA Foundation Project(No.2014MS-001), National Key Basic Research 
Development Program (973 Program) (No.2012CB518104) and Military Medical Key foundation of 12th five-year plan (No.BWS11C061).

\section{Disclosure Statement}

The authors have declared that no competing interests exist.

\section{Reference}

1 Rahimi S: Squamous cell carcinoma of skin: A brief review. Clin Ter 2013;164:143-147.

2 Winkelman C: Inactivity and inflammation: Selected cytokines as biologic mediators in muscle dysfunction during critical illness. AACN clinical issues 2004;15:74-82.

3 Ye H, Wu Q, Zhu Y, Guo C, Zheng X: Ginsenoside rh2 alleviates dextran sulfate sodium-induced colitis via augmenting tgfbeta signaling. Mol Biol Rep 2014;41:5485-5490.

4 Kikuchi Y, Sasa H, Kita T, Hirata J, Tode T, Nagata I: Inhibition of human ovarian cancer cell proliferation in vitro by ginsenoside rh2 and adjuvant effects to cisplatin in vivo. Anticancer Drugs 1991;2:63-67.

5 Tode T, Kikuchi Y, Hirata J, Kita T, Imaizumi E, Nagata I: [inhibitory effects of oral administration of ginsenoside rh2 on tumor growth in nude mice bearing serous cyst adenocarcinoma of the human ovary]. Nihon Sanka Fujinka Gakkai zasshi 1993;45:1275-1282.

6 Tode T, Kikuchi Y, Kita T, Hirata J, Imaizumi E, Nagata I: Inhibitory effects by oral administration of ginsenoside rh2 on the growth of human ovarian cancer cells in nude mice. J Cancer Res Clin Oncol 1993;120:24-26.

7 Nakata H, Kikuchi Y, Tode T, Hirata J, Kita T, Ishii K, Kudoh K, Nagata I, Shinomiya N: Inhibitory effects of ginsenoside rh2 on tumor growth in nude mice bearing human ovarian cancer cells. Jpn J Cancer Res 1998;89:733-740.

8 Tang XP, Tang GD, Fang CY, Liang ZH, Zhang LY: Effects of ginsenoside rh2 on growth and migration of pancreatic cancer cells. World J Gastroenterol 2013;19:1582-1592.

9 Liu J, Shimizu K, Yu H, Zhang C, Jin F, Kondo R: Stereospecificity of hydroxyl group at c-20 in antiproliferative action of ginsenoside rh2 on prostate cancer cells. Fitoterapia 2010;81:902-905.

10 Li B, Zhao J, Wang CZ, Searle J, He TC, Yuan CS, Du W: Ginsenoside rh2 induces apoptosis and paraptosislike cell death in colorectal cancer cells through activation of p53. Cancer Lett 2011;301:185-192.

11 Oh M, Choi YH, Choi S, Chung H, Kim K, Kim SI, Kim DK, Kim ND: Anti-proliferating effects of ginsenoside rh2 on mcf-7 human breast cancer cells. Int J Oncol 1999;14:869-875.

12 Choi S, Kim TW, Singh SV: Ginsenoside rh2-mediated g1 phase cell cycle arrest in human breast cancer cells is caused by p15 ink4b and p27 kip1-dependent inhibition of cyclin-dependent kinases. Pharm Res 2009;26:2280-2288.

13 Li S, Gao Y, Ma W, Guo W, Zhou G, Cheng T, Liu Y: Egfr signaling-dependent inhibition of glioblastoma growth by ginsenoside rh2. Tumour Biol 2014;35:5593-5598.

14 Xu J, Fan W, Tu XX, Zhang T, Hou ZJ, Guo T, Shu X, Luo X, Liu Y, Peng F, Wang C, Xu L, Zhou H, Liu Q: Neural ganglioside $\mathrm{gd} 2(+)$ cells define a subpopulation of mesenchymal stem cells in adult murine bone marrow. Cell Physiol Biochem 2013;32:889-898.

15 Petersson M, Niemann C: Stem cell dynamics and heterogeneity: Implications for epidermal regeneration and skin cancer. Curr Med Chem 2012;19:5984-5992.

16 Perez-Losada J, Balmain A: Stem-cell hierarchy in skin cancer. Nat Rev Cancer 2003;3:434-443.

17 Chen Q, Cai ZK, Chen YB, Gu M, Zheng DC, Zhou J, Wang Z: Poly r(c) binding protein-1 is central to maintenance of cancer stem cells in prostate cancer cells. Cell Physiol Biochem 2015;35:1052-1061.

18 He QZ, Luo XZ, Wang K, Zhou Q Ao H, Yang Y, Li SX, Li Y, Zhu HT, Duan T: Isolation and characterization of cancer stem cells from high-grade serous ovarian carcinomas. Cell Physiol Biochem 2014;33:173-184.

19 Nagata T, Sakakura C, Komiyama S, Miyashita A, Nishio M, Murayama Y, Komatsu S, Shiozaki A, Kuriu Y, Ikoma H, Nakanishi M, Ichikawa D, Fujiwara H, Okamoto K, Ochiai T, Kokuba Y, Sonoyama T, Otsuji E: Expression of cancer stem cell markers cd133 and cd44 in locoregional recurrence of rectal cancer. Anticancer Res 2011;31:495-500.

20 Fang DD, Kim YJ, Lee CN, Aggarwal S, McKinnon K, Mesmer D, Norton J, Birse CE, He T, Ruben SM, Moore PA: Expansion of cd133(+) colon cancer cultures retaining stem cell properties to enable cancer stem cell target discovery. Br J Cancer 2010;102:1265-1275. 
21 Shi C, Tian R, Wang M, Wang X, Jiang J, Zhang Z, Li X, He Z, Gong W, Qin R: Cd44+ cd133+ population exhibits cancer stem cell-like characteristics in human gallbladder carcinoma. Cancer Biol Ther 2010;10:1182-1190.

22 Ottaiano A: Finding markers for cancer stem cells in renal cell carcinoma: Looking beyond cd133. Cell Cycle 2010;9:4431.

23 Ma S, Chan KW, Lee TK, Tang KH, Wo JY, Zheng BJ, Guan XY: Aldehyde dehydrogenase discriminates the cd133 liver cancer stem cell populations. Mol Cancer Res 2008;6:1146-1153.

24 Wang J, Sakariassen PO, Tsinkalovsky O, Immervoll H, Boe SO, Svendsen A, Prestegarden L, Rosland G, Thorsen F, Stuhr L, Molven A, Bjerkvig R, Enger PO: Cd133 negative glioma cells form tumors in nude rats and give rise to cd133 positive cells. Int J Cancer 2008;122:761-768.

25 Takubo K, Ohmura M, Azuma M, Nagamatsu G, Yamada W, Arai F, Hirao A, Suda T: Stem cell defects in atmdeficient undifferentiated spermatogonia through DNA damage-induced cell-cycle arrest. Cell Stem Cell 2008;2:170-182.

26 Duester G: Families of retinoid dehydrogenases regulating vitamin a function: Production of visual pigment and retinoic acid. Eur J Biochem 2000;267:4315-4324.

27 Magni M, Shammah S, Schiro R, Mellado W, Dalla-Favera R, Gianni AM: Induction of cyclophosphamideresistance by aldehyde-dehydrogenase gene transfer. Blood 1996;87:1097-1103.

28 Silva IA, Bai S, McLean K, Yang K, Griffith K, Thomas D, Ginestier C, Johnston C, Kueck A, Reynolds RK, Wicha MS, Buckanovich RJ: Aldehyde dehydrogenase in combination with cd133 defines angiogenic ovarian cancer stem cells that portend poor patient survival. Cancer Res 2011;71:3991-4001.

29 Ma I, Allan AL: The role of human aldehyde dehydrogenase in normal and cancer stem cells. Stem Cell Rev 2011;7:292-306.

30 Liu Y, Jiang X, Zeng Y, Zhou H, Yang J, Cao R: Proliferating pancreatic beta-cells upregulate aldh. Histochem Cell Biol 2014;142:685-691.

31 Zhang L, Wang L, Liu X, Zheng D, Liu S, Liu C: Aldh expression characterizes g1-phase proliferating beta cells during pregnancy. PLoS One 2014;9:e96204.

32 Kinzel B, Pikiolek M, Orsini V, Sprunger J, Isken A, Zietzling S, Desplanches M, Dubost V, Breustedt D, Valdez R, Liu D, Theil D, Muller M, Dietrich B, Bouwmeester T, Ruffner H, Tchorz JS: Functional roles of lgr4 and lgr5 in embryonic gut, kidney and skin development in mice. Dev Biol 2014;390:181-190.

33 Haegebarth A, Clevers H: Wnt signaling, lgr5, and stem cells in the intestine and skin. Am J Pathol 2009;174:715-721.

34 Liu S, Gong Z, Chen M, Liu B, Bian D, Wu K: Lgr5-positive cells are cancer stem cells in skin squamous cell carcinoma. Tumour Biol 2014;35:11605-11612.

35 Yin YC, Lin CC, Chen TT, Chen JY, Tsai HJ, Wang CY, Chen SY: Clozapine induces autophagic cell death in nonsmall cell lung cancer cells. Cell Physiol Biochem 2015;35:945-956.

36 Zhao M, Sun L, Yu XJ, Miao Y, Liu JJ, Wang H, Ren J, Zang WJ: Acetylcholine mediates ampk-dependent autophagic cytoprotection in h9c2 cells during hypoxia/reoxygenation injury. Cell Physiol Biochem 2013;32:601-613.

37 Wei YM, Li X, Xu M, Abais JM, Chen Y, Riebling CR, Boini KM, Li PL, Zhang Y: Enhancement of autophagy by simvastatin through inhibition of rac1-mtor signaling pathway in coronary arterial myocytes. Cell Physiol Biochem 2013;31:925-937.

38 Lin R, Feng J, Dong S, Pan R, Zhuang H, Ding Z: Regulation of autophagy of prostate cancer cells by betacatenin signaling. Cell Physiol Biochem 2015;35:926-932.

39 Kim W, Kim M, Jho EH: Wnt/beta-catenin signalling: From plasma membrane to nucleus. Biochem J 2013;450:9-21.

40 Gewirtz DA: Autophagy and senescence in cancer therapy. J Cell Physiol 2014;229:6-9.

41 Gao C, Cao W, Bao L, Zuo W, Xie G, Cai T, Fu W, Zhang J, Wu W, Zhang X, Chen YG: Autophagy negatively regulates wnt signalling by promoting dishevelled degradation. Nat Cell Biol 2010;12:781-790.

42 Zhang Y, Wang F, Han L, Wu Y, Li S, Yang X, Wang Y, Ren F, Zhai Y, Wang D, Jia B, Xia Y, Chang Z: Gabarapl1 negatively regulates wnt/beta-catenin signaling by mediating dvl2 degradation through the autophagy pathway. Cell Physiol Biochem 2011;27:503-512.

43 Cicchini M, Chakrabarti R, Kongara S, Price S, Nahar R, Lozy F, Zhong H, Vazquez A, Kang Y, Karantza V: Autophagy regulator becn 1 suppresses mammary tumorigenesis driven by wht 1 activation and following parity. Autophagy 2014;10:2036-2052.

44 Holla S, Kurowska-Stolarska M, Bayry J, Balaji KN: Selective inhibition of ifng-induced autophagy by mir155- and mir31-responsive wnt5a and shh signaling. Autophagy 2014;10:311-330. 